\title{
DOS ESPECIES NUEVAS DE BURSERA (BURSERACEAE) DEL ESTADO DE OAXACA (MÉXICO) ${ }^{1}$
}

\author{
JERZY RZEDOWSKI \\ Y \\ Graciela Calderón de Rzedowski \\ Instituto de Ecología, A.C. \\ Centro Regional del Bajío \\ Apartado postal 386 \\ 61600 Pátzcuaro, Michoacán
}

\section{RESUMEN}

Se describen como nuevas y se ilustran Bursera heliae y $B$. isthmica, especies aparentemente restringidas en su distribución al sureste de Oaxaca. La primera está vinculada con $B$. cuneata (Schlecht.) Engl., mientras que la segunda se relaciona con B. glabra (Jacq.) Tr. \& Planch. y con B. ribana Rzed. \& Calderón. Se incluye asimismo una enumeración de 30 especies adicionales de Bursera conocidas hasta la fecha para el mencionado estado.

Palabras clave: Bursera, Burseraceae, México, Oaxaca.

\begin{abstract}
Bursera heliae and $B$. isthmica are described as new and illustrated. The distribution of both seems to be restricted to the southeastern part of the Mexican state of Oaxaca. The first one is related to B. cuneata (Schlecht.) Engl., while the second is linked with B. glabra (Jacq.) Tr. \& Planch. and with B. ribana Rzed. \& Calderón. A list of additional 30 species of Bursera known from Oaxaca is included.
\end{abstract}

Key words: Bursera, Burseraceae, México, Oaxaca.

El bosque tropical caducifolio ocupaba y aún ocupa importantes extensiones en el estado de Oaxaca. Es característico de superficies cerriles de la región conocida como La Cañada, que cubre las porciones más secas y calientes de la cuenca alta del Papaloapan. En otros tiempos este tipo de vegetación prosperaba también en muchos parajes de la porción conocida como Valles Centrales, que es la más densamente

1 Trabajo realizado con apoyo económico del Instituto de Ecología, A.C. (cuenta 902-03), del Consejo Nacional de Ciencia y Tecnología y de la Comisión Nacional para el Conocimiento y Uso de la Biodiversidad. 
poblada del estado y en la mayor parte de la cual ya sólo quedan vestigios de la cubierta vegetal original. No falta tampoco en los sectores inferiores de la porción oaxaqueña de la cuenca del Balsas ni en la del Río Verde y es de los más característicos de la cuenca del río Tehuantepec. Se extiende en amplias superficies de la región istmeña y de allí a todo lo largo de la costa pacífica, pero en la mitad occidental de la misma ya ha sufrido una gran mella.

Aunque los árboles del género Bursera constituyen un componente substancial de los bosques tropicales caducifolios de todas estas partes de Oaxaca, el conocimiento de su identidad deja aún bastante que desear. En la actualidad existen para el estado los registros relativamente confiables de los siguientes 30 taxa:

Bursera aloexylon (Schiede ex Schlecht.) Engl.

(¿incluyendo Elaphrium longipedunculatum Rose?)

Bursera aptera Ramírez

Bursera arida (Rose) Standl.

Bursera ariensis (H.B.K.) Mcv. \& Rzed.

Bursera aspleniifolia Brandegee

Bursera bicolor (Willd. ex Schlecht.) Engl.

Bursera biflora (Rose) Standl.

Bursera bipinnata (DC.) Engl.

Bursera bolivarii Rzed.

Bursera cinerea Engl.

Bursera copallifera (DC.) Bullock

Bursera discolor Rzed.

Bursera excelsa (H.B.K.) Engl.

Bursera fagaroides (H.B.K.) Engl.

Bursera galeottiana Engl.

Bursera glabrifolia (H.B.K.) Engl.

Bursera graveolens (H.B.K.) Tr. \& Planch.

Bursera heteresthes Bullock

Bursera instabilis McV. \& Rzed.

Bursera krusei Rzed.

Bursera lancifolia (Schlecht.) Engl.

Bursera laurihuertae Rzed. \& Calderón

Bursera longipes (Rose) Standl.

Bursera mirandae C. Toledo

Bursera morelensis Ramírez

Bursera schlechtendalii Engl.

Bursera simaruba (L.) Sarg.

Bursera submoniliformis Engl.

Bursera vejar-vazquezii Miranda

Bursera xochipalensis Rzed.

Varias especies más se han registrado para Oaxaca, pero su presencia real está sujeta a una comprobación ulterior. Indudablemente existe un contingente importante de 
taxa adicionales, de los que no pocos están todavía por descubrirse, sobre todo en la región costera y en la parte correspondiente a la cuenca del Balsas.

De otros ya se cuenta con colectas, inclusive en algunos casos relativamente antiguas, pero los materiales disponibles a menudo no resultan suficientes para decidir sobre su correcta ubicación taxonómica. Las exploraciones de años recientes han ayudado en forma significativa al esclarecimiento de varios de estos casos inciertos, dos de los cuales se presentan a continuación en calidad de especies nuevas para el conocimiento, ambas pertenecientes a la sección Bullockia.

\section{Bursera heliae Rzedowski \& Calderón sp. n.}

Arbor vel interdum frutex usque $8 \mathrm{~m}$ altus ut videtur dioecius; truncus cortice grisaceo non exfolianti, ramuli juniores dense puberuli et minutissime glanduloso-puberuli; foliorum rosulae juventute cataphyllis exterioribus mature deciduis cinctae, folia trifoliolata et imparipinnata, rhachidi anguste alata, foliola 3-5(7) plerumque ovata usque obovata, suborbicularia vel oblonga, $0.8-4.5 \mathrm{~cm}$ longa, $0.7-3.5 \mathrm{~cm}$ lata (foliolum terminale plerumque lateralibus valde majus), apice rotundata usque acuta, basi cuneata usque truncata, marginibus duplo serratis, utrinque puberula, minutissime glanduloso-puberula et mollia, supra rugoso-bullata, subtus manifeste nervata, coriacea; inflorescentiae racemosopaniculatae, flores masculi tetrameri, calycis lobi lineares vel lineari-lanceolati ca. $2 \mathrm{~mm}$ longi, petala albida vel flavida elliptica vel anguste obovata ca. $3 \mathrm{~mm}$ longa, stamina 8; flores feminei masculorum similes sed calycis lobis longioribus, ovarium biloculare, stigmata 2; drupae orbiculari-ovoideae ca. $10 \mathrm{~mm}$ diametro, pyrenae ovoideae ca. $7 \mathrm{~mm}$ longae pseudoarillo flavo-aurantiaco per 5/6 partibus inferis indutae.

Árbol o a veces arbusto hasta de $8 \mathrm{~m}$ de alto y tronco hasta de $30 \mathrm{~cm}$ de diámetro, aparentemente dioico; corteza gris, no exfoliante, con abundante oleoresina aromática; ramas rojizas oscuras, ramillas jóvenes densamente pubérulas y además con numerosos pelos glandulares diminutos; hojas con frecuencia aglomeradas en rosetas sobre ramillas cortas, otras veces alternas en ramillas de crecimiento nuevo, precedidas en su aparición por un conjunto de catafilos lineares de 5 a $16 \mathrm{~mm}$ de largo y 0.5 a $1 \mathrm{~mm}$ de ancho, densamente pubérulos por fuera, precozmente caducos, hojas trifolioladas e imparipinnadas, hasta de $7 \mathrm{~cm}$ de largo y $5.5 \mathrm{~cm}$ de ancho, peciolo hasta de $3 \mathrm{~cm}$ de largo, densamente hírtulo-pubérulo y con muy diminutos pelos glandulares, raquis (de estar presente) con alas poco manifiestas, de margen entero, por lo general de menos de $1 \mathrm{~mm}$ de ancho de cada lado, foliolos 3 ó 5 , rara vez 7 , sésiles o subsésiles, comúnmente ovados, pero variando a obovados, suborbiculares u oblongos, de 0.8 a $4.5 \mathrm{~cm}$ de largo y de 0.7 a $3.5 \mathrm{~cm}$ de ancho, el terminal con frecuencia notablemente más grande que los laterales, redondeados o a veces obtusos o agudos en el ápice, cuneados a truncados en la base, margen por lo común doblemente aserrado (con los dientes escotados en el ápice) o al menos con esta tendencia visible, dientes principales 8 a 15(20) de cada lado, pubérulos y suaves al tacto y con presencia adicional de diminutos pelos glandulares en ambas superficies, verde-grisáceos oscuros en el haz, más claros en el envés, de textura coriácea, en la madurez manifiestamente ampuloso-rugosos en el haz y con las nervaduras muy prominentes en el envés; inflorescencias racimosas o a veces paniculadas, con frecuencia desarrollándose al mismo tiempo que las hojas 


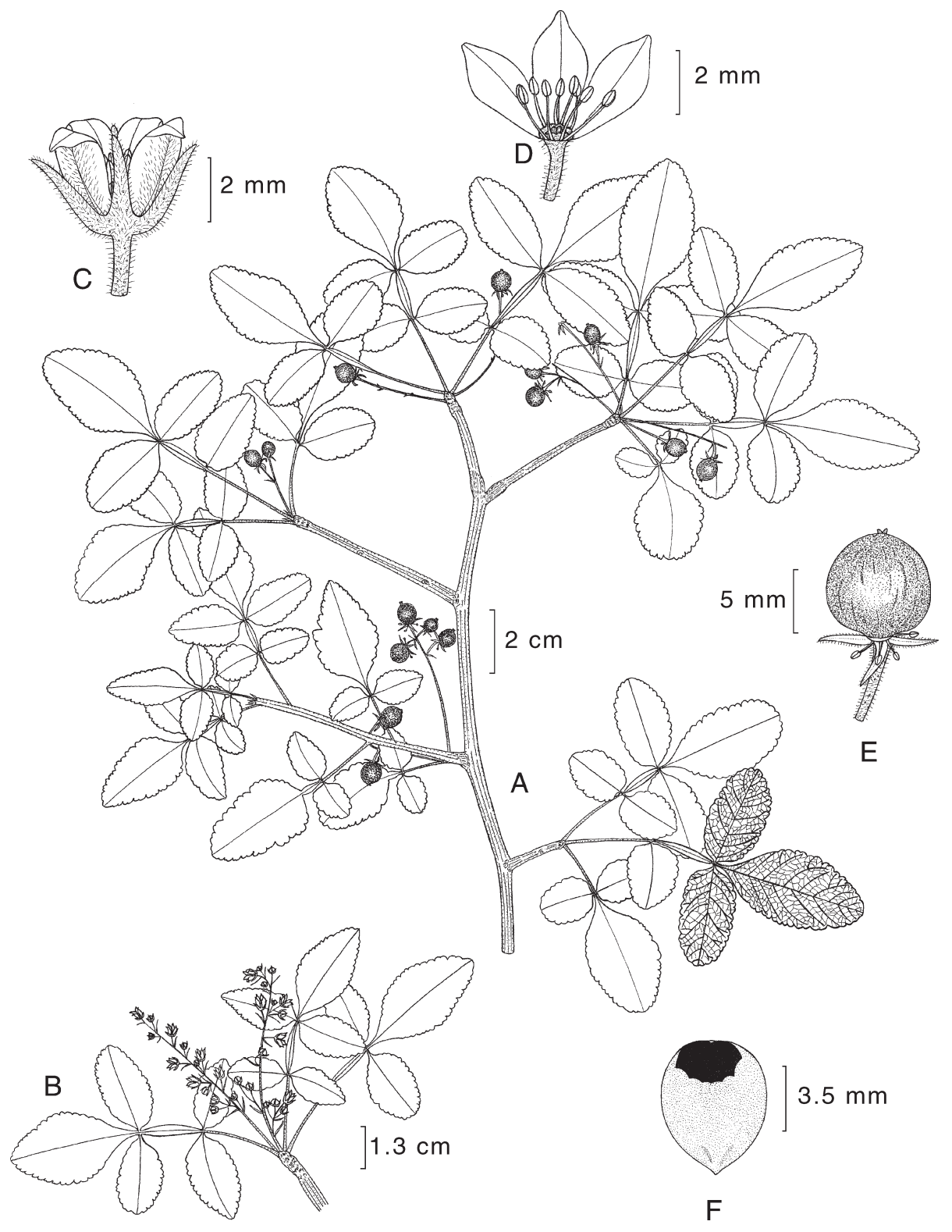

Bursera heliae Rzed. \& Calderón. A. rama con hojas y frutos; B. rama con flores masculinas; C. flor masculina en vista externa; D. flor masculina desprovista del cáliz y de un pétalo; E. fruto; F. hueso. llustrado por Rogelio Cárdenas. 
tiernas, bracteadas, sus ejes densamente hírtulo-pubérulos y con presencia de diminutos pelos glandulares; flores de ambos sexos tetrámeras; inflorescencias masculinas hasta de $6 \mathrm{~cm}$ de largo, a veces ramificadas, brácteas lineares, de ca. $3 \mathrm{~mm}$ de largo, pedicelos hasta de $3 \mathrm{~mm}$ de largo, cáliz dividido casi hasta la base, sus lóbulos lineares a linearlanceolados, de ca. $2.5 \mathrm{~mm}$ de largo, densamente hírtulo-pubérulos por fuera, pétalos blanquecinos a amarillentos, elípticos a angostamente obovados, de $3 \mathrm{a} 4 \mathrm{~mm}$ de largo, agudos y ligeramente apiculados en el ápice, pubérulos por fuera, disco en forma de cojín, 8-lobulado, estambres 8, anteras oblongas, de ca. $1 \mathrm{~mm}$ de largo, gineceo diminuto, casi ausente; inflorescencias femeninas hasta de $5 \mathrm{~cm}$ de largo, por lo general sin ramificarse y con pocas flores, pedicelos hasta de $2 \mathrm{~mm}$ de largo, flores femeninas similares a las masculinas, pero con los segmentos del cáliz de ca. $3.5 \mathrm{~mm}$ de largo, estaminodios con anteras de ca. $0.8 \mathrm{~mm}$ de largo, ovario bilocular, estigmas 2; drupas orbicular-ovoides, de ca. $8 \mathrm{~mm}$ de diámetro, rojas en la madurez, glabras, hueso ovoide, de ca. $7 \mathrm{~mm}$ de largo, cubierto en sus $5 / 6$ partes por un pseudoarilo amarillentoanaranjado, la porción expuesta negra.

TIPO: México, Oaxaca: Portillo de San Dionisio, $13 \mathrm{~km}$ al SSE de Matatlán, distrito de Tlacolula, matorrales secundarios, alt. 1,700 m, 10.VIII.1976, J. Rzedowski 34385 (holotipo IEB, isotipos por distribuirse).

Material adicional examinado: México, Oaxaca: $6.5 \mathrm{~km}$ al E de Mitla, exposición SW, camino a Sta. Ma. Albarradas, municipio de Mitla, H. Arroyo 112 (IEB); Río Grande, municipio de Mitla, F. Ventura 16346 (IEB); $5 \mathrm{~km}$ al SW de San Antonio Albarradas (camino a Mitla, cañada de orientación SE-NW), municipio de Albarradas, H. Arroyo 91 (IEB); Hierve El Agua, springs SW of San Lorenzo Albarradas, S of road between Mitla and Totontepec, municipio de Albarradas, distrito de Tlacolula, $16^{\circ} 52^{\prime} \mathrm{N}, 96^{\circ} 17^{\prime} \mathrm{W}, R$. E. Gereau et al. 2033 (IEB, OAX); La Chivitoa, paraje de San Pedro Taviche, municipio de San Pedro Taviche, L. Servín et al. s. n., 19.VIII.2001 (IEB); San Jerónimo Taviche, municipio de San Jerónimo Taviche, distrito de Ocotlán, C. Robles 53 (IEB, OAX); cerro El Copalito, San Nicolás Yaxe, M. Ambosio et al. 626 (IEB); alrededores de San Pedro Quiatoni, COTECOCA brigada XVI 16 (IEB); $5 \mathrm{~km}$ de la desv. Portillo S. Dionisio Totolapan, municipio de Totolapan, distrito de Tlacolula, A. Saynes 1270 (IEB, OAX); $10 \mathrm{~km}$ desv. Portillo S. Dionisio - Totolapan, municipio de Totolapan, distrito de Tlacolula, A. Saynes 1282 (IEB, OAX); $4 \mathrm{~km}$ al W de Totolapan, municipio de Totolapan, distrito de Tlacolula, A. Flores 1357 (IEB, OAX); a 4 km de Totolapan, rumbo a Oaxaca, municipio de Totolapan, distrito de Tlacolula, A. Flores 1322 (IEB, OAX); $6.1 \mathrm{~km}$ al E de Totolapan, carr. Oaxaca - Tehuantepec, municipio de Totolapan, distrito de Tehuantepec, R. Torres y C. Martínez 8999 (MEXU); barranca Las Calabazas, $3 \mathrm{~km}$ al W de Totolapan, camino al Duraznillo, municipio de Totolapan, distrito de Tlacolula, S. Acosta 984 (IEB, OAX); El Derrumbadero, municipio de Totolapan, F. Ventura 16531 (IEB); 4 km al NE de San Juanico, sobre el camino a Tehuantepec, municipio de Totolapan, J. Rzedowski 33032 (IEB); Santa María Zoquitlán, municipio de Santa María Zoquitlán, distrito de Tlacolula, C. Robles 89 (IEB); 1 km al NE de Santa María Zoquitlán, municipio de Santa María Zoquitlán, distrito de Tlacolula, A. Flores 1395 (IEB, OAX); Mano de León, municipio de Santa María Zoquitlán, F. Ventura 16580 (IEB); 4.5 km NNW, municipio de San Juan Mixtepec, distrito de Miahuatlán, 16¹9'08" N, 96¹8'08" W, E. Hunn OAX-1121(MEXU). 
B. heliae se restringe en su distribución a la porción de altitudes medias de la cuenca del río Tehuantepec, en el sector centro-suroriental del estado de Oaxaca, donde prospera en elevaciones entre 850 y 1,800 m s.n.m., formando parte del bosque tropical caducifolio.

En virtud de sus foliolos doblemente dentados e inflorescencias relativamente cortas, $B$. heliae queda ubicada en el grupo 4 del esquema clasificatorio de McVaugh y Rzedowski (1965). Su parentesco más cercano parece dirigirse a B. cuneata (Schlecht.) Engl., taxon conocido del centro de México, cuya área de distribución llega a Puebla y Guerrero, pero que no se ha colectado en Oaxaca. Esta especie comparte con $B$. heliae el carácter de hojas rugoso-ampulosas en el haz, así como diversos rasgos de flores y frutos, pero se distingue en sus foliolos más numerosos ((3)5 a 9(13)), de forma más bien oblonga y pubescencia tomentosa en el envés, así como en los catafilos más grandes.

Se han recogido para $B$. heliae los siguientes nombres comunes: copal blanco, copal colorado, copal tecomaca, tecomaca, tecomacal, yàg-yàal (lengua zapoteca). Se registró asimismo el uso de su oleoresina en forma de incienso y también su empleo medicinal en algunas comunidades zapotecas.

El epíteto de la especie nueva se consagra como homenaje póstumo a la recientemente desaparecida Dra. Helia Bravo Hollis, destacada botánica mexicana, dedicada en forma especial al conocimiento de la familia Cactaceae.

\section{Bursera isthmica Rzedowski \& Calderón sp. n.}

Arbor vel interum frutex usque $8 \mathrm{~m}$ altus ut videtur dioecius; truncus cortice grisaceo non exfolianti, ramuli juniores dense puberuli, foliorum rosulae juventute cataphyllis exterioribus mature deciduis cinctae; folia imparipinnata, rhachis manifeste alata, foliola (5)7-13(15), ovata vel subrhombea, 1.5-4.5(7) cm longa, 1-2(3.5) cm lata (foliolum termi-nale plerumque lateralibus aliquantum majus), apice obtusa vel rotundata, raro acuta, basi cuneata usque truncata, marginibus grosse et plerumque irregulariter serratis, saepe sublobatis, supra puberula, subtus densius puberula et mollia, chartaceo-coriacea; inflorescentiae racemoso-paniculatae; flores masculi tetrameri puberuli, calycis lobi lineari-subulati ca. $2 \mathrm{~mm}$ longi, petala alba lanceolato-elliptica ca. $3 \mathrm{~mm}$ longa, stamina 8; flores feminei masculorum similes, ovarium biloculare, stigmata 2; drupae orbiculari-ovoideae 6-7 $\mathrm{mm}$ diametro, pyrenae biconvexae pseudoarillo flavido-aurantiaco per 2/3 partibus inferis indutae.

Árbol o arbusto hasta de $8 \mathrm{~m}$ de alto, con tronco hasta de $35 \mathrm{~cm}$ de diámetro, aparentemente dioico; corteza gris, no exfoliante, con abundante oleoresina aromática, ramillas jóvenes densamente pubérulas; hojas frecuentemente aglomeradas en rosetas en los ápices de ramillas cortas, otras veces alternas en ramillas de crecimiento nuevo, precedidas en su aparición por un conjunto de catafilos triangulares, de ca. $3 \mathrm{~mm}$ de largo, agudos en el ápice, manifiestamente cóncavo-convexos (acucharados), rígidos y coriáceos, densamente pubérulos por fuera, pronto caedizos, hojas imparipinnadas, hasta de $23 \mathrm{~cm}$ de largo y $10 \mathrm{~cm}$ de ancho, peciolo hasta de $6.5 \mathrm{~cm}$ de largo, densamente pubérulo, raquis con alas manifiestas de 1 a $2.5 \mathrm{~mm}$ de ancho de cada lado, con el margen entero, incrementando con frecuencia su anchura hacia la parte distal del intersticio, peciólulos de 0 a $1.5 \mathrm{~mm}$ de largo, foliolos (5)7 a 13(15), ovados 
Rzedowski y Calderón de Rzedowski: Dos Especies Nuevas de Bursera de Oaxaca

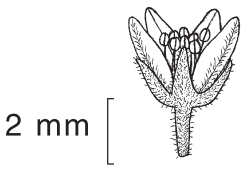

B

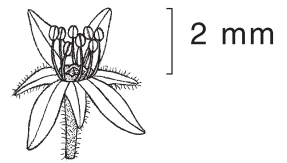

C

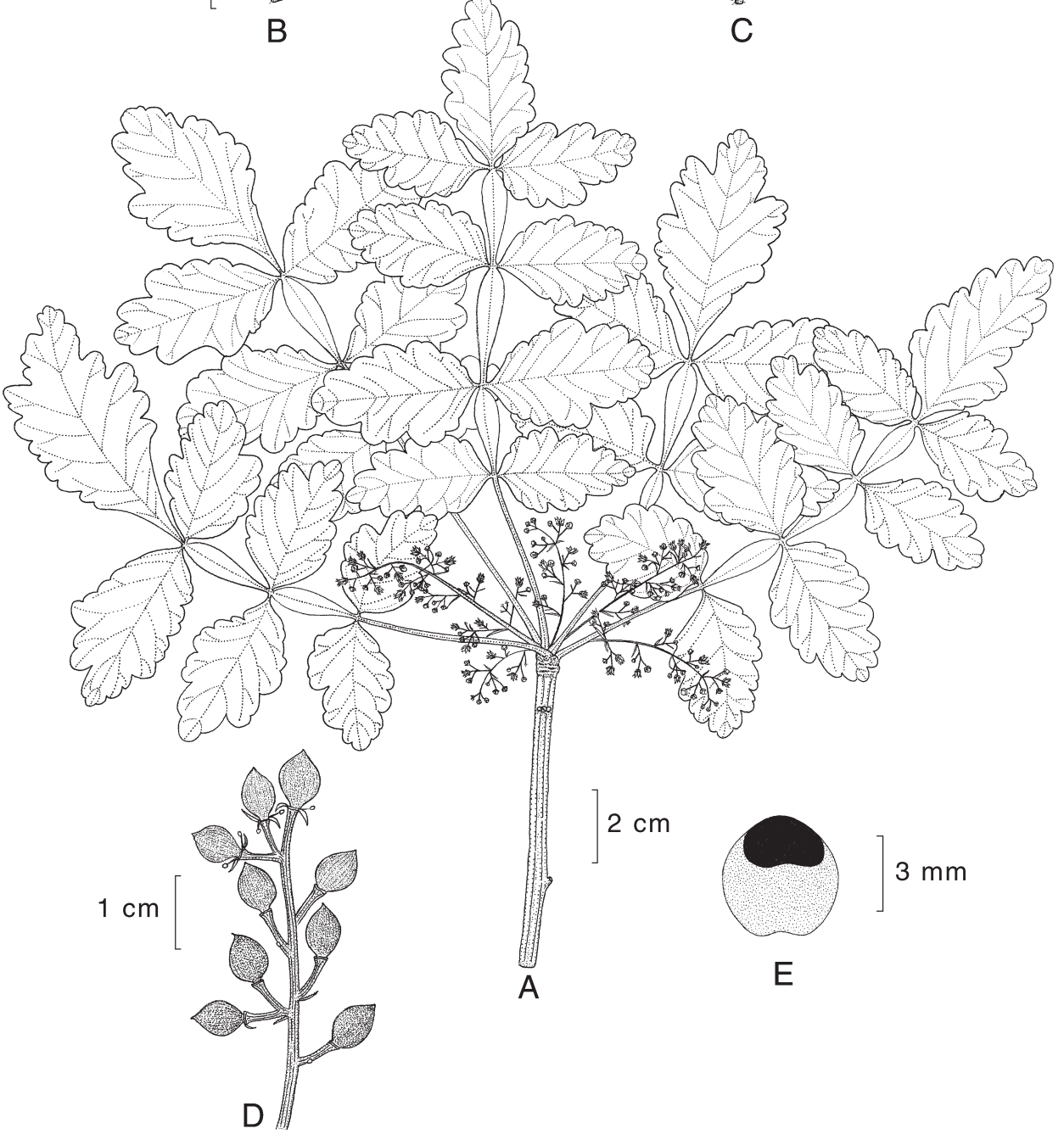

Bursera isthmica Rzed. \& Calderón. A. rama con hojas e inflorescencias masculinas; B. flor masculina en vista externa; C. flor masculina artificialmente abierta; D. infrutescencia; $\mathrm{E}$. hueso. llustrado por Rogelio Cárdenas. 
a subrómbicos, de 1.5 a $4.5(7) \mathrm{cm}$ de largo y 1 a 2(3.5) $\mathrm{cm}$ de ancho, el terminal por lo general un poco más largo que los laterales, ápice obtuso a redondeado, rara vez agudo, base cuneada a truncada, con frecuencia algo oblicua, margen toscamente serrado, a menudo en forma irregular y con algunos dientes mucho más grandes y profundos que otros y a su vez algo serrados, insinuando la tendencia a la formación de lóbulos, dientes principales 3 a 7 de cada lado, redondeados o a veces agudos en el ápice, pubérulos en el haz con pelos de base ampulosa, mucho más densamente pubérulos y suaves al tacto en el envés, de color verde olivo en el haz, más pálidos en el envés, de textura cartáceo-coriácea; inflorescencias racimoso-paniculadas, a menudo presentes al mismo tiempo que las hojas tiernas, bracteadas, sus ejes densamente pubérulos; flores de ambos sexos tetrámeras; inflorescencias masculinas hasta de $9(15) \mathrm{cm}$ de largo, con frecuencia ramificadas pero no muy abiertas, brácteas linear-subuladas, de ca. $2 \mathrm{~mm}$ de largo, pedicelos hasta de $2.5 \mathrm{~mm}$ de largo, cáliz dividido casi hasta la base, sus lóbulos linear-subulados, de ca. $2 \mathrm{~mm}$ de largo, densamente pubérulos por fuera, pétalos blancos, lanceolado-elípticos, de ca. $3 \mathrm{~mm}$ de largo, esparcidamente pubérulo-hispídulos por fuera, disco en forma de cojín inconspicuamente 8-lobulado, estambres 8, anteras oblongas, de ca. $0.4 \mathrm{~mm}$ de largo, gineceo vestigial; inflorescencias femeninas hasta de $6 \mathrm{~cm}$ de largo, por lo general no ramificadas, pedicelos hasta de $1.5 \mathrm{~mm}$ de largo en flor, flores femeninas similares a las masculinas pero un poco más pequeñas, estaminodios con anteras de ca. $0.3 \mathrm{~mm}$ de largo, ovario bilocular, estigmas 2; drupas orbicular-ovoides, de 6 a $7 \mathrm{~mm}$ de diámetro, diminutamente apiculadas, glabras, hueso biconvexo, de 4 a $5 \mathrm{~mm}$ de largo, cubierto en sus $2 / 3$ inferiores por un pseudoarilo amarillento-anaranjado, la parte expuesta negra.

TIPO: México, Oaxaca: cerro Cuachi, al O de Salina Cruz, municipio de Salina Cruz, distrito de Tehuantepec, selva baja caducifolia, 30.VIII.1985, C. Martínez R.175 (IEB, isotipo en MEXU).

Material adicional examinado: México, Oaxaca: Tapanalá, $1.5 \mathrm{~km} \mathrm{NE}$, municipio de San Pedro Huamelula, distrito de Tehuantepec, 1558'23" N, 9543'33" W, J. Rivera 1658 (IEB, MEXU); Tapanalá, $500 \mathrm{~m}$ al NW de la población, municipio de San Pedro Huamelula, distrito de Tehuantepec, 1598'15" N, 9543'22" W, M. Elorsa 3493 (IEB), 3494 (IEB); km 244 por la Calzada de Piedra, carr. Tehuantepec - Oaxaca, municipio de Tehuantepec, $C$. Martínez R. 748 (IEB, MEXU); lado N del cerro Pozo Zorrillo, 6 km al NO de Tehuantepec, municipio de Tehuantepec, 16²2' N, 95¹6' W, C. Martínez R. 1054 (IEB, MEXU); rancho Ricardo, al N de Buenos Aires, entrando por Hierba Santa, municipio de Tehuantepec, distrito de Tehuantepec, R. Torres y C. Martínez 7308 (MEXU); San Vicente Mazatán, $20 \mathrm{~km}$ al $\mathrm{N}$ del Morro, entrando por el crucero de Santa Clara, municipio de Tehuantepec, $16^{\circ} 07^{\prime} \mathrm{N}$, 95²5' W, C. Martínez R. 1562 (IEB, MEXU); Tehuantepec, T. MacDougall 17 (MEXU); ibid., E. Matuda 2204 (MEXU), 9244 (MEXU); al pie del cerro Tecuani, al O de Salina Cruz, municipio de Salina Cruz, distrito de Tehuantepec, C. Martínez R. 244 (IEB, MEXU); en el camino Carnero a Rincón Bamba, Salina Cruz, municipio de Salina Cruz, distrito de Tehuantepec, R. Torres 8765 (IEB); 2 miles north of La Ventosa, 2 miles east of Salina Cruz, R. McVaugh 21839 (ENCB); 12.5 miles east of Juchitán, along the Pan-American highway between Oaxaca and Tuxtla Gutiérrez, R. McVaugh 21851 (ENCB). 
El nombre de la especie se relaciona con su área de distribución restringida, pues todo parece indicar que sólo existe en el extremo sur del Istmo de Tehuantepec, donde habita sobre laderas de cerros de altitudes inferiores a $200 \mathrm{~m}$, como componente del bosque tropical caducifolio y recibe los nombres comunes de copal y coyul. Se emplea para integrar cercas vivas y también como medicinal.

En este contexto cabe enfatizar el hecho de que tanto Lorence y García-Mendoza (1989), como Pérez-García et al. (2001) identifican al sector meridional del Istmo como área de concentración de endemismos en lo que concierne a plantas vasculares.

Como ya lo adelantaron los autores del presente trabajo (Rzedowski y Calderón de Rzedowski 2000: 55), en la forma de sus hojas y frutos $B$. isthmica presenta semejanzas con B. ribana Rzed. \& Calderón, taxon conocido de la región costera de Jalisco y Michoacán, pero esta última difiere en ser planta mucho menos pubescente, de hojas con foliolos más pequeños (de (6)10 a 20(25) mm de largo y 6 a $16 \mathrm{~mm}$ de ancho), inflorescencias más abiertas y lóbulos del cáliz anchamente triangulares, de ca. $0.5 \mathrm{~mm}$ de largo. Otra especie probablemente muy emparentada es B. glabra (Jacq.) Tr. \& Planch., conocida de Colombia, y a cuya sinonimia sometió también Cuatrecasas (1957: 429) a B. howellii Standl., descrita de Costa Rica y Colombia. De esta planta no se han visto más que descripciones y fotografías de ejemplares, de las que cabe deducir que con toda verosimilitud pertenece al mismo linaje, pero difiere de $B$. isthmica en sus hojas y foliolos más pequeños, estos últimos (según Cuatrecasas, op. cit.) de 6 a $24 \mathrm{~mm}$ de largo por 6 a $18 \mathrm{~mm}$ de ancho y en los lóbulos del cáliz lanceolado-triangulares. Tanto este último autor (op. cit.) como Engler (1883: 52) indican también que las flores de B. glabra son pentámeras, pero es factible que se trate de un error.

En $B$. isthmica tampoco se han observado hojas francamente bipinnadas, que con cierta frecuencia se presentan en $B$. glabra y en $B$. ribana. Sin embargo, en muchos ejemplares de la primera cabe notar una definida tendencia a la formación de lóbulos en los márgenes de los foliolos. Es tan manifiesto este carácter que ha sido la causa de la identificación de algunos especímenes como $B$. diversifolia Rose, nombre que se ha estado empleando para híbridos entre B. bipinnata (DC.) Engl. y algunos otros miembros de la sección Bullockia, como B. copallifera (DC.) Bullock, B. cuneata (Schlecht.) Engl., B. excelsa (H.B.K.) Engl., B. palmeri S. Wats. y B. tomentosa (Jacq.) Tr. \& Planch.

El estudio de los ya relativamente amplios materiales de $B$. isthmica revela que se trata de una especie bien definida, quizá de un antiguo origen híbrido, en el que puede haber intervenido B. bipinnata, pero con la cual no está conviviendo en la actualidad.

\section{AGRADECIMIENTOS}

Damos las gracias a la Biól. Remedios Aguilar, encargada del herbario OAX y a la Biól. Silvia Salas, presidenta de SERBO (Sociedad para el Estudio de los Recursos Bióticos de Oaxaca, A.C.) por habernos proporcionado importantes materiales de herbario, así como extractos de sus bases de datos. Agradecemos también a la Biól. Felisa Herrador, responsable de la biblioteca del Instituto de Ecología, A.C., por su ayuda en la obtención de literatura pertinente. 


\section{LITERATURA CITADA}

Cuatrecasas, J. 1957. Prima flora colombiana. I. Burseraceae. Webbia 12: 375-441.

Engler, A. 1883. Burseraceae. DC. Monogr. Phaner. 4: 1-169.

Lorence, D. H. y A. García-Mendoza. 1989. Oaxaca, Mexico. In: D. G. Campbell y H. D. Hammond (eds.). Floristic inventory of tropical countries. New York Botanical Garden. Nueva York. pp. 253-269.

McVaugh, R. y J. Rzedowski. 1965. Synopsis of the genus Bursera in western Mexico, with notes on the material of Bursera collected by Sessé \& Mociño. Kew Bull. 18: 317-382.

Pérez-García, E. A., J. Meave y C. Gallardo. 2001. Vegetación y flora de la región de Nizanda, Istmo de Tehuantepec, México. Acta Bot. Mex. 56: 19-88.

Rzedowski, J. y G. Calderón de Rzedowski. 2000. Tres especies nuevas de Bursera (Burseraceae) de la región costera del occidente de México. Acta Bot. Mex. 50: 47-59. 